\title{
Spinning strings as small black rings
}

\section{Atish Dabholkar and Ashik Iqubal}

Tata Institute of Fundamental Research

Homi Bhabha Road, Mumbai, 400 005, India

E-mail: atish@theory.tifr.res.in, iqubal@tifr.res.in

\section{Norihiro lizuka}

Kavli Institute for Theoretical Physics, University of California,

Santa Barbara, CA 93106-4030, U.S.A.

E-mail: iizuka@kitp.ucsb.edu

\section{Ashoke Sen}

Harish-Chandra Research Institute

Chhatnag Road, Jhusi, Allahabad 211019, India

E-mail: sen@mri.ernet.in

\section{Masaki Shigemori}

California Institute of Technology

452-48, Pasadena, CA 91125, U.S.A.

E-mail: shige@theory.caltech.edu

ABSTRACT: Certain supersymmetric elementary string states with spin can be viewed as small black rings whose horizon has the topology of $S^{1} \times S^{d-3}$ in a $d$-dimensional string theory. By analyzing the singular black ring solution in the supergravity approximation, and using various symmetries of the $\alpha^{\prime}$ corrected effective action we argue that the BekensteinHawking-Wald entropy of the black string solution in the full string theory agrees with the statistical entropy of the same system up to an overall normalization constant. While the normalization constant cannot be determined by the symmetry principles alone, it can be related to a similar normalization constant that appears in the expression for small black holes without angular momentum in one less dimension. Thus agreement between statistical and macroscopic entropy of $(d-1)$-dimensional non-rotating elementary string states would imply a similar agreement for a $d$-dimensional elementary string state with spin. Our analysis also determines the structure of the near horizon geometry and provides us with a geometric derivation of the Regge bound. These studies give further evidence that a ring-like horizon is formed when large angular momentum is added to a small black hole.

KeYwords: Black Holes in String Theory, Superstrings and Heterotic Strings. 


\section{Contents}

1. Introduction and summary 1

2. A brief review of the microscopic counting 3

3. Scaling analysis for black rings

4. Entropy function and near horizon geometry 11

5. Discussion 18

A. Supergravity small black rings in general dimensions 20

\section{Introduction and summary}

Recently, there has been great deal of progress in computing corrections to black hole entropy due to the effect of higher derivative terms in string theory effective action and comparing the answer to the statistical entropy of the same system [1-13]. A particularly interesting class of examples is provided by the stringy 'small' black holes. These are singular solutions of the classical supergravity equations of motion since they have vanishing area of the event horizon. However microscopically they are described by BPS states of the fundamental string and hence have non-zero degeneracies. A simple class of such examples is provided by the FP system or the so-called Dabholkar-Harvey (DH) system [14], which is obtained by winding a fundamental heterotic string $-w$ times around a circle $S^{1}$ and putting $n$ units of momentum along the same circle. If the right-movers are in the ground state then such a state is BPS but it can carry arbitrary left-moving oscillations. ${ }^{1}$ Its microscopic entropy is given by

$$
S_{\text {micro }}=4 \pi \sqrt{n w} \text {. }
$$

Although in the supergravity approximation the corresponding solution has zero horizon area and hence zero entropy, one expects that the result will be modified by the higher derivative corrections since close to the (singular) horizon the curvature and other field strengths become strong and the supergravity approximation is expected to break down. However one finds that the string coupling constant near the horizon is small and hence one

\footnotetext{
${ }^{1}$ In the literature one often finds two different sets of conventions. The first one uses the convention that for a BPS state the momentum and winding along $S^{1}$ will have the same sign. The second one uses the convention that for a BPS state the momentum and winding along $S^{1}$ will have opposite sign. Here we are using the second convention.
} 
need not worry about string loop corrections. By analyzing the behavior of the supergravity solution near the horizon and using various symmetries of the full string tree level effective action one finds that the $\alpha^{\prime}$-corrected entropy must have the form

$$
C \sqrt{n w}
$$

for some constant $C$ 15-18. However, the constant $C$ cannot be calculated based on symmetry principles alone. Recently for the special case of four dimensional small black holes this constant was calculated in [9] using a special class of higher derivative terms in the effective action and was found to give the value $4 \pi$ in precise agreement with the microscopic answer. Later it was demonstrated in [19-21] that other higher derivative corrections do not change the result. Furthermore, it was shown that using special ensembles to define entropy, the agreement between microscopic and macroscopic counting can be pushed beyond the leading order to all orders in the large charge expansion [9, 22 - 24].

The next natural question is what will happen if we add angular momentum $J$ to the system. Studying microscopic states and making use of 4D-5D connection [25-30], it was argued in [31] that if large angular momentum is added to a small black hole in five dimensions with horizon topology $S^{3}$, it will turn into a small black ring whose horizon has topology of $S^{1} \times S^{2}$. The size of $S^{2}$ is of the order of the string scale, and hence is small compared with the typical horizon scales of ordinary supersymmetric black ring solutions in [32- 35] for which supergravity approximation is good. Furthermore, in [31], it was shown that the entropy of small black rings can be related to that of four dimensional small black holes, ${ }^{2}$ and that the entropy calculation based on this argument matches the independent entropy calculation of the rotating DH system by Russo and Susskind [39] (see also 40, 41, 25, 19, 42]).

Since a ring-like horizon is expected to show up for large $J$, it is interesting to study the near horizon geometry of small black rings from a macroscopic, geometric point of view. In particular we would like to know the $J$ dependence of the horizon geometry. One would also like to generalize the results to higher dimensions. These are the problems we shall address in this paper. In particular we show that in arbitrary dimensions the supergravity equations of motion admit a singular black ring solution carrying the same charge quantum numbers as that of a rotating elementary string. This solution is characterized by the charges $n$ and $w$ introduced earlier, the angular momentum $J$ and a dipole charge $Q$ that represents how many times the fundamental string winds in the azimuthal directions. The microscopic entropy of such a system can be easily computed from the spectrum of elementary string states with spin and gives the answer

$$
S_{\text {stat }}=4 \pi \sqrt{n w-J Q} .
$$

These states exist only for $n w \geq J Q$, which can be regarded as the Regge bound for BPS states. On the other hand since the supergravity solution is singular we cannot directly calculate the macroscopic entropy. Nevertheless by examining the solution in a region of low curvature, where supergravity approximation is expected to be valid, we find that the

\footnotetext{
${ }^{2}$ Using the fuzzball picture [36, 37] it was also argued in 38] that this system becomes a black ring.
} 
space-time associated with the solution develops closed time-like curves for $n w<J Q$ [43]. Thus absence of such curves requires $n w>J Q$, thereby providing a geometric derivation of the Regge bound. ${ }^{3}$ Furthermore, by using a symmetry argument very similar to that in 15-18] we show that the entropy, if non-zero, is given by

$$
S_{\mathrm{BH}}=C \sqrt{n w-J Q},
$$

for some constant $C$. Furthermore $C$ is the same constant that would appear in the expression (1.2) for the macroscopic entropy of a non-rotating black hole in one less dimension. Thus agreement between microscopic and macroscopic entropy of a $(d-1)$-dimensional non-rotating small black hole would imply a similar agreement for $d$-dimensional rotating small black rings. This analysis also determines the near horizon geometry in terms of some unknown constants of order unity. In particular we can estimate the sizes of various circles and spheres near the horizon.

The rest of the paper is organized as follows. In section 2 we briefly review the computation of the statistical entropy of rotating elementary string states leading to the answer (1.3). In section 3 we describe the supergravity solution describing the rotating small black ring and study the effect of higher derivative corrections to this solution, eventually arriving at the formula (1.4) for the black ring entropy. This analysis also determines the dependence of the near horizon geometry on various charges in terms of some unknown universal functions of the radial coordinate. In section 1 we use the entropy function formalism [45] and scaling arguments for the case of ring-like horizons, and show that the results are consistent with the ones derived in section 3. This analysis determines the near horizon geometry in terms of some unknown constants of order unity. We conclude in section 5 with a discussion of our results and some open issues.

\section{A brief review of the microscopic counting}

Let us consider heterotic string theory in $\mathbf{R}_{t} \times \mathbf{R}^{d-1} \times S^{1} \times T^{9-d}$ with $4 \leq d \leq 9$ with $\mathbf{R}_{t} \times \mathbf{R}^{d-1}$ denoting $d$-dimensional Minkowski space. Our objects of interest are the BPS elementary string excitations in this theory carrying $n$ units of momentum and $-w$ units of winding charge along the circle $S^{1}$ and angular momentum $J$ in a two dimensional plane of $\mathbf{R}^{d-1}$. In the Ramond sector such states are obtained by acting with left-moving oscillators of level $N=n w+1$ on the Fock vacuum carrying charges $(n, w)$. In the NS sector we also need to act on the Fock vacuum by a right-moving fermionic oscillator of level $1 / 2$. Let $x^{1}$ and $x^{2}$ denote the coordinates of the two dimensional plane in which the state carries angular momentum $J$. Then if we define $x^{ \pm}=\left(x^{1} \pm i x^{2}\right) / \sqrt{2}$ and denote by $\alpha_{-m}^{ \pm}$the corresponding oscillators of level $m$, then the quantum number $J$ is given by the difference between the number of $\alpha_{-m}^{+}$and the number of $\alpha_{-m}^{-}$oscillators acting on the Fock vacuum. The statistical entropy is then given by the logarithm of the number of elementary string states subject to the restriction that the number of $\alpha_{-m}^{+}$oscillators minus the number of $\alpha_{-m}^{-}$oscillators acting on the Fock vacuum is precisely $J$.

\footnotetext{
${ }^{3}$ For oscillating string solutions describing string states with angular momentum, a derivation of the Regge bound was given earlier in 44 by requiring regularity of the source terms in the equation of motion.
} 
In our analysis we shall examine a finer definition of the statistical entropy of rotating elementary strings introduced in [46]. Let $Q$ be a positive integer. Let us further assume, for definiteness, that $J$ is positive. We now count all states of the form

$$
\left(\alpha_{-Q}^{+}\right)^{J} \mathcal{O}|v a c\rangle
$$

where $\mathcal{O}$ is an arbitrary combination of left-moving oscillators of level $N_{\mathcal{O}}=n w-Q J+1$, and $|v a c\rangle$ denotes the Fock vacuum for Ramond sector states, and the Fock vacuum acted on by a right-moving fermionic oscillator of level $1 / 2$ for the NS sector states. The statistical entropy of such states can be easily computed and gives the answer 46]

$$
S_{\text {stat }} \simeq 4 \pi \sqrt{N_{\mathcal{O}}} \simeq 4 \pi \sqrt{n w-J Q}
$$

for large $n w-J Q$. Note that as defined, $\mathcal{O}$ can have any spin and hence the states which are counted by this procedure do not have definite angular momentum. However the dominant contribution to the entropy defined this way comes from the states for which $\mathcal{O}$ has spin close to zero, i.e. states with spin close to $J$. Thus even if we restrict $\mathcal{O}$ to have total spin zero the result for entropy would have the form given in (2.2) at the leading order.

By restricting to states of the form (2.1) we have restricted to an exponentially small subset of all the states carrying angular momentum $J$. In particular the dominant contribution to the statistical entropy for a fixed $J$ comes from states where most of the angular momentum is created by the $\alpha_{-1}^{+}$oscillators, i.e. from the states with $Q=1$. The reason for considering states of the form (2.1) however is that we can distinguish states with different $Q$ by the fields they produce even though $Q$ is not a conserved charge. In particular the operator $\alpha_{-Q}^{+}$creates quanta of a mode for which the space-time coordinates $x^{1}$ and $x^{2}$ have $Q$ complete oscillations as we go once around the string. Thus a state of the form (2.1) where large number of these modes condense should correspond to a configuration where the projection of the string in the $x^{1}-x^{2}$ plane has $Q$ units of winding around its center of mass coordinate. As a result we expect that the corresponding gravity solution will be represented by a ring like structure in the $x^{1}-x^{2}$ plane with $Q$ units of winding charge along the ring. As we shall see, the entropy of the corresponding solution will match the statistical entropy given in eq. (2.2).

\section{Scaling analysis for black rings}

In this section we shall study the geometry of a small black ring in arbitrary dimensions. Although in the supergravity approximation this solution is singular along the ring, by studying carefully the geometry near the singularity we shall be able to determine the dependence of the entropy on various charges assuming that the higher derivative corrections modify the solution in a way such that it has a finite entropy. This will essentially involve generalization of the scaling analysis of [15-18] to the case of rotating black rings.

Consider heterotic string theory in $\mathbf{R}_{t} \times \mathbf{R}^{d-1} \times S^{1} \times T^{9-d}$ with $4 \leq d \leq 9$. Since in our analysis the moduli associated with the torus $T^{9-d}$ will be frozen completely, and furthermore all the mixed components of the metric and the anti-symmetric tensor field 
with one leg along $T^{9-d}$ and another leg along one of the other directions will be set to zero, it will be more convenient to regard the theory as a theory in $(d+1)$ space-time dimensions. ${ }^{4}$ The massless fields in $(d+1)$ space-time dimensions that are relevant for analyzing the black ring solution are the string metric $G_{\mu \nu}$, the anti-symmetric tensor field $B_{\mu \nu}$ and dilaton $\Phi_{d+1}$. The string tree level action involving these fields has the form

$$
\mathcal{S}=\int d^{d+1} x \sqrt{-\operatorname{det} G} e^{-2 \Phi_{d+1}} \mathcal{L},
$$

where $\mathcal{L}$ is a function of the metric, Riemann tensor, the 3 -form field strength

$$
H_{\mu \nu \rho}=\partial_{\mu} B_{\nu \rho}+\text { cyclic permutations of } \mu, \nu, \rho+\Omega_{\mu \nu \rho}^{\mathrm{CS}, \mathrm{L}}(G),
$$

covariant derivatives of these quantities, as well as covariant derivatives of the dilaton $\Phi_{d+1}$ but not of $\Phi_{d+1}$ itself. Here $\Omega_{\mu \nu \rho}^{\mathrm{CS}, \mathrm{L}}(G)$ denotes the Lorentz Chern-Simons 3-form constructed out of the string metric $G_{\mu \nu}$ - more precisely out of the spin connection compatible with this metric - with some appropriate coefficient. Since we set all the gauge fields in $(d+1)$-dimensions to zero, there are no gauge Chern-Simons term in the definition of $H_{\mu \nu \rho}$. In the supergravity approximation where we have only two derivative terms, the Lorentz Chern-Simons term disappears from the expression for $H_{\mu \nu \rho}$ and $\mathcal{L}$ takes the form

$$
\mathcal{L}=\frac{1}{(2 \pi)^{d-2}\left(\alpha^{\prime}\right)^{(d-1) / 2}}\left[R_{G}+4 G^{\mu \nu} \partial_{\mu} \Phi \partial_{\nu} \Phi-\frac{1}{12} G^{\mu \mu^{\prime}} G^{\nu \nu^{\prime}} G^{\rho \rho^{\prime}} H_{\mu \nu \rho} H_{\mu^{\prime} \nu^{\prime} \rho^{\prime}}\right] .
$$

We denote the coordinates of $\mathbf{R}_{t}$ and $S^{1}$ by $t$ and $x^{d}$ respectively. Let the coordinate radius of $x^{d}$ direction be $R_{d}$. For $\mathbf{R}^{d-1}$ we use a special coordinate system in which the flat metric on $\mathbf{R}^{d-1}$ takes the form:

$$
d \mathbf{x}_{d-1}^{2}=\frac{R^{2}}{(x-y)^{2}}\left[\frac{d y^{2}}{y^{2}-1}+\left(y^{2}-1\right) d \psi^{2}+\frac{d x^{2}}{1-x^{2}}+\left(1-x^{2}\right) d \Omega_{d-4}^{2}\right],
$$

where $d \Omega_{d-4}$ denotes the line elements on the $(d-4)$-sphere, $R$ is a constant whose value is given in (3.8), and $x, y$ take values in the range

$$
-1 \leq x \leq 1, \quad-\infty<y \leq-1 .
$$

The relationship between these coordinates and the cartesian coordinates of $\mathbf{R}^{d-1}$ has been given in eqs. (A.8), (A.22).

A black ring solution in the supergravity approximation, describing a rotating fundamental string of the type described in section 2, has been constructed in appendix A based on [47, 44, 48, 49] and takes the form:

$$
\begin{aligned}
d s_{s t r, d+1}^{2} & =f_{f}^{-1}\left[-\left(d t-A_{i} d x^{i}\right)^{2}+\left(d x^{d}-A_{i} d x^{i}\right)^{2}+\left(f_{p}-1\right)\left(d t-d x^{d}\right)^{2}\right]+d \mathbf{x}_{d-1}^{2} \\
e^{2 \Phi_{d+1}} & =g^{2} f_{f}^{-1}, \quad B_{t d}=-\left(f_{f}^{-1}-1\right), \quad B_{t i}=-B_{d i}=f_{f}^{-1} A_{i},
\end{aligned}
$$

\footnotetext{
${ }^{4}$ In other words we consider a solution for which the world-sheet theory of the fundamental string propagating in this background is a direct sum of two conformal field theories. The first one is a free field theory associated with the coordinates of $T^{9-d}$ and the sixteen additional left-moving world-sheet bosons of the heterotic string theory. The second one is an interacting theory associated with the string propagation in the $(d+1)$-dimensional black ring solution. Our focus will be on the latter theory.
} 
where $i=1,2, \ldots, d-1$,

$$
\begin{aligned}
f_{f} & =1+\frac{Q_{f}}{R^{d-3}}\left(\frac{x-y}{-2 y}\right)^{(d-3) / 2}{ }_{2} F_{1}\left(\frac{d-3}{4}, \frac{d-1}{4} ; 1 ; 1-\frac{1}{y^{2}}\right), \\
f_{p} & =1+\frac{Q_{p}}{R^{d-3}}\left(\frac{x-y}{-2 y}\right)^{(d-3) / 2}{ }_{2} F_{1}\left(\frac{d-3}{4}, \frac{d-1}{4} ; 1 ; 1-\frac{1}{y^{2}}\right), \\
A_{i} d x^{i} & =-\frac{d-3}{2} \frac{q}{R^{d-5}} \frac{\left(y^{2}-1\right)(x-y)^{(d-5) / 2}}{(-2 y)^{(d-1) / 2}}{ }_{2} F_{1}\left(\frac{d-1}{4}, \frac{d+1}{4} ; 2 ; 1-\frac{1}{y^{2}}\right) d \psi,
\end{aligned}
$$

and $Q_{f}, Q_{p}, q$ and $R$ are related to the quantized charges $n, w, Q$ and the angular momentum $J$ via the relations

$$
\begin{aligned}
q & =\frac{16 \pi G_{d}}{(d-3) \Omega_{d-2} \alpha^{\prime}} Q, & R^{2} & =\alpha^{\prime} \frac{J}{Q}, \\
Q_{f} & =\frac{16 \pi G_{d} R_{d}}{(d-3) \Omega_{d-2} \alpha^{\prime}} w, & Q_{p} & =\frac{16 \pi G_{d}}{(d-3) \Omega_{d-2} R_{d}} n .
\end{aligned}
$$

Here $\Omega_{D}$ is the area of unit $D$-sphere and $G_{d}$ is the $d$-dimensional Newton's constant obtained by regarding the $S^{1}$ direction as compact

$$
16 \pi G_{d}=\frac{16 \pi G_{d+1}}{2 \pi R_{d}}=\frac{(2 \pi)^{d-3} g^{2} \alpha^{\prime(d-1) / 2}}{R_{d}} .
$$

$n$ and $-w$ denote respectively the number of units of momentum and winding charge along the $S^{1}$ direction labeled by $x^{d}$. $Q$ represents the number of units of winding charge along the singular ring situated at $y=-\infty$. From the perspective of an asymptotic observer $Q$ appears as a dipole charge and does not represent a conserved gauge charge. In order that the metric given in (3.6) has the standard signature, we require $R^{2}>0$, i.e.

$$
J Q>0
$$

For $d=5$ the hypergeometric functions simplify and we have

$$
f_{f}=1+\frac{Q_{f}(x-y)}{2 R^{2}}, \quad f_{p}=1+\frac{Q_{p}(x-y)}{2 R^{2}}, \quad A_{i} d x^{i}=-\frac{q}{2}(1+y) d \psi .
$$

This $d=5$ solution can be obtained by setting to zero one of the three charges and two of the three dipoles charges of the supersymmetric black ring solution of [32-35]. The solution with general $d$ can also be found by U-dualizing the supergravity supertube solution of 44].

For reasons that will become clear later, we shall work with the following assignment of charges:

$$
J \gg Q \gg 1, \quad n \sim w, \quad n w \sim J Q, \quad 1-\frac{J Q}{n w} \sim 1 .
$$

Since the ring singularity occurs as $y \rightarrow-\infty$ we shall study the geometry near the singularity by examining the large negative $y$ region. For

$$
\frac{R}{\sqrt{\alpha^{\prime}}} \gg|y| \gg\left(\frac{1}{g^{2} Q}\right)^{\frac{1}{d-4}} \frac{R}{\sqrt{\alpha^{\prime}}},\left(\frac{R_{d}^{2}}{g^{2} Q}\right)^{\frac{1}{d-4}} \frac{R}{\sqrt{\alpha^{\prime}}}, 1,
$$


the functions $f_{f}, f_{p}$ and $A_{\psi}$ take the form:

$$
\begin{gathered}
f_{f} \simeq \begin{cases}c_{d} Q_{f} R^{3-d}|y|^{d-4} & \text { for } d>4, \\
\frac{1}{\pi} Q_{f} R^{-1} \log |y| & \text { for } d=4,\end{cases} \\
f_{p} \simeq \begin{cases}c_{d} Q_{p} R^{3-d}|y|^{d-4} & \text { for } d>4, \\
\frac{1}{\pi} Q_{p} R^{-1} \log |y| & \text { for } d=4,\end{cases} \\
A_{\psi} \simeq \begin{cases}-c_{d} q R^{5-d}|y|^{d-4} & \text { for } d>4, \\
-\frac{1}{\pi} q R \log |y| & \text { for } d=4,\end{cases}
\end{gathered}
$$

where

$$
c_{d}=\frac{\Gamma\left(\frac{d-4}{2}\right)}{2 \sqrt{\pi} \Gamma\left(\frac{d-3}{2}\right)} .
$$

Let us restrict to the case $d \geq 5$ and use the convention $\alpha^{\prime}=1$. Using eqs. (3.8) and $(3.9)$ we see that in the region (3.14) the original solution (3.6) takes the form:

$$
\begin{aligned}
d s_{s t r, d+1}^{2}= & \frac{n}{w} \frac{1}{R_{d}^{2}}\left(d x^{d}-d t\right)^{2}+2 \frac{(d-3) \Omega_{d-2}}{c_{d}(2 \pi)^{d-3}} \frac{1}{w} \frac{R}{g^{2}}\left(-\frac{R}{y}\right)^{d-4} d t\left(d x^{d}-d t\right) \\
& +2 \frac{J}{w R_{d}} d \psi\left(d x^{d}-d t\right)+R^{2} \frac{d y^{2}}{y^{4}}+R^{2} d \psi^{2}+\left(-\frac{R}{y}\right)^{2} d \Omega_{d-3}^{2} \\
\frac{1}{2} B_{\mu \nu} d x^{\mu} \wedge d x^{\nu}= & -\frac{(d-3) \Omega_{d-2}}{c_{d}(2 \pi)^{d-3}} \frac{1}{w} \frac{R}{g^{2}}\left(-\frac{R}{y}\right)^{d-4} d t \wedge\left(d x^{d}-d t\right) \\
& +\frac{J}{w R_{d}} d\left(x^{d}-t\right) \wedge d \psi+\mathrm{constant} \\
e^{2 \Phi_{d+1}}= & \frac{(d-3) \Omega_{d-2}}{c_{d}(2 \pi)^{d-3}} \frac{R}{w}\left(-\frac{R}{y}\right)^{d-4}
\end{aligned}
$$

where

$$
d \Omega_{d-3}^{2} \equiv \frac{d x^{2}}{1-x^{2}}+\left(1-x^{2}\right) d \Omega_{d-4}^{2}
$$

is the squared line element on a $(d-3)$-sphere and the constant in the expression for $B$ represents a constant 2-form proportional to $d t \wedge d x^{d}$ which can be removed by gauge transformation. For this solution all scalars constructed out of curvatures and other field strengths are small in the region (3.13). For example the $(d+1)$-dimensional Ricci scalar in the string frame goes as $(y / R)^{2}$ in this region. As a result the supergravity approximation is still valid in this region and the form of the solution (3.16) can be trusted.

It is easy to see from (3.16) that the $2 \times 2$ matrix describing the metric in the $\psi-x^{d}$ plane develops a negative eigenvalue for $J Q>n w$. Since the $\psi-x^{d}$ plane is topologically a two dimensional torus, the corresponding space-time has closed time-like curves. Thus absence of closed time-like curve requires that

$$
J Q \leq n w .
$$

Thus is precisely the Regge bound. The fact that (3.18) can be derived by requiring absence of closed time-like curves was noted in [43] in a different U-duality frame. Here 
we see that this geometrical condition is identical to the Regge bound in the spectrum of elementary BPS string states that follows from the left-right level matching condition in the microscopic theory analyzed in section 2 .

Note that both the conditions $J Q>0$ given in (3.10) and the Regge bound (3.18) are expected from the profile of the microscopic string underlying the solution as discussed in the appendix. This construction led to a manifestly positive $J Q$ and $(n w-J Q)$. The point however is that once we have obtained the solution, we can interpret it as a solution of the supergravity equations of motion parametrized by the charges $J, Q, n$ and $w$ without worrying about where it came from. Nevertheless regularity of the space-time geometry requires both the conditions $(3.10)$ and $(3.18)$ to be satisfied.

From now on we shall restrict our analysis to the case $J Q<n w$. By examining the solution (3.16) we see that this seems to depend on the various charges $n, w, J, Q$ as well as the asymptotic values $g$ and $R_{d}$ of the moduli fields. We shall now show that by making a suitable coordinate transformation the solution can be made to be independent of the parameters $g$ and $R_{d}$, and have simple dependence on the charges. We define

$$
\begin{aligned}
\sigma & =\sqrt{\frac{n}{w}-\frac{J Q}{w^{2}}} \frac{1}{R_{d}}\left(x^{d}-t\right), \quad \rho=-\frac{R}{y}, \\
\tau & =\frac{(d-3) \Omega_{d-2}}{c_{d}(2 \pi)^{d-3}} \frac{R_{d}}{\sqrt{n w-J Q}} \frac{R}{g^{2}} t, \quad \chi=\sqrt{\frac{J}{Q}} \psi+\frac{\sqrt{J Q}}{w} \frac{1}{R_{d}}\left(x^{d}-t\right),
\end{aligned}
$$

In this coordinate system the region (3.13) gets mapped to

$$
1 \ll \rho \ll\left(g^{2} Q\right)^{\frac{1}{d-4}},\left(g^{2} Q / R_{d}^{2}\right)^{\frac{1}{d-4}}, R,
$$

and the coordinates $\chi$ and $\sigma$ have the following periods:

$$
(\sigma, \chi) \equiv\left(\sigma, \chi+2 \pi \sqrt{\frac{J}{Q}}\right) \equiv\left(\sigma+2 \pi \sqrt{\frac{n}{w}} \sqrt{1-\frac{J Q}{n w}}, \chi+2 \pi \frac{\sqrt{J Q}}{w}\right) .
$$

The $\sigma-\chi$ plane at fixed values of the other coordinates is topologically a two dimensional torus of coordinate area

$$
A_{\sigma \chi}=4 \pi^{2} \sqrt{\frac{J n}{Q w}} \sqrt{1-\frac{J Q}{n w}} .
$$

In terms of the coordinates (3.19) the field configuration given in (3.16) in the region 3.20 ) takes the form:

$$
\begin{aligned}
d s_{s t r, d+1}^{2} & =d \sigma^{2}+d \chi^{2}+2 \rho^{d-4} d \tau d \sigma+d \rho^{2}+\rho^{2} d \Omega_{d-3}^{2} \\
\frac{1}{2} B_{\mu \nu} d x^{\mu} \wedge d x^{\nu} & =-\rho^{d-4} d \tau \wedge d \sigma+\left(\frac{n w}{J Q}-1\right)^{-1 / 2} d \sigma \wedge d \chi \\
e^{2 \Phi_{d+1}} & =\frac{(d-3) \Omega_{d-2}}{c_{d}(2 \pi)^{d-3}} \frac{1}{w} \sqrt{\frac{J}{Q}} \rho^{d-4} .
\end{aligned}
$$

By examining the solution we see that in this coordinate system the solution as well as the periodicities of the $(\sigma, \chi)$ coordinates are independent of the parameters $g$ and $R_{d}$. Furthermore the dependence of the solution on the charges comes via some additive constants 
in the expressions for $B_{\sigma \chi}$ and $\Phi_{d+1}$, and in the periodicities of the $(\sigma, \chi)$ plane. We shall make use of this observation later to determine how the $\alpha^{\prime}$-corrected solution depends on the charges.

We now note the following properties of this background:

- For $\rho \gg 1$ curvature and other field strengths associated with this configuration are small. Hence the higher derivative corrections to the equations of motion are negligible. Since $J \sim n w / Q \sim w^{2} / Q$ and $Q \gg 1$, the string coupling constant $e^{\Phi_{d+1}}$ is also small in this region showing that the string loop corrections are also negligible.

- For $\rho \sim 1$ the curvature and other field strengths become of order unity and hence the $\alpha^{\prime}$ corrections become important. However $e^{\Phi_{d+1}}$ continues to be small since for the choice of charges of the form (3.12), $\frac{1}{w} \sqrt{\frac{J}{Q}} \sim \frac{1}{Q}$ is small. Thus string loop corrections are not important.

- If we naively put a 'stretched horizon' at $\rho \sim 1$, and calculate the naive entropy from the area of the stretched horizon, spanned by $\chi, \sigma$ and the coordinates of $S^{d-3}$ we get an answer proportional to $\sqrt{n w-Q J}$. This seems to agree with the formula (2.2) for the statistical entropy. However we should keep in mind that at $\rho \sim 1$ higher derivative corrections are important and neither the form of the solution nor the Bekenstein-Hawking formula for the entropy can be trusted. This is the problem to which we shall now turn.

The analysis of higher derivative corrections to the solution given in (3.23) is facilitated by the following observations:

i) The solution given in (3.23) is independent of the coordinates $\tau, \sigma$ and $\chi$. It also has a $\mathrm{SO}(d-2)$ spherical symmetry acting on the coordinates of the unit $(d-3)$-sphere whose line element has been denoted by $d \Omega_{d-3}$. We expect that the $\alpha^{\prime}$-corrected solution will also preserve these symmetries.

ii) Since the string coupling constant at the horizon is small, we can ignore string loop corrections to the effective action. The tree level $\alpha^{\prime}$ corrected theory can be described by an effective Lagrangian density in $(d+1)$ dimensions which does not depend on the periods of the $\sigma$ and the $\chi$ coordinates. Since we are looking for solutions which are independent of the $\sigma$ and $\chi$ coordinates, the independence of the Lagrangian density on their periodicities guarantees that there is no dependence of the solution on the periodicities of these variables. ${ }^{5}$ In particular the solution will have precisely the same form even if the $\sigma$ and the $\chi$ coordinates had been non-compact.

iii) By examining the form of the solution $(3.23)$ we see that except for an additive constant term $\left(\frac{n w}{J Q}-1\right)^{-1 / 2}$ in the expression for $B_{\sigma \chi}$ and an additive term of the form

\footnotetext{
${ }^{5}$ One could worry about possible corrections to the effective action due to world-sheet instantons wrapping the $\chi-\sigma$ torus. However such contributions will be exponentially suppressed due to large area of this torus measured in the string metric.
} 
$\frac{1}{2} \ln \left(\frac{1}{w} \sqrt{\frac{J}{Q}}\right)$ in the expression for $\Phi_{d+1}$, the solution is independent of the various charges and the asymptotic values of the various moduli e.g. $g, R_{d}$ etc. Since the tree level effective Lagrangian density depends on $\Phi_{d+1}$ only via an overall multiplicative factor of $e^{-2 \Phi_{d+1}}$ and terms involving derivatives of $\Phi_{d+1}$ and depends on $B_{\mu \nu}$ only through its field strength $d B, B_{\mu \nu}$ and $\Phi_{d+1}$ can be shifted by arbitrary constants without affecting the rest of the solution. Thus we shall expect that even after including the $\alpha^{\prime}$ corrections the solution continues to be independent of the various charges and the parameters $g, R_{d}$ etc. except for an additive factor of $\frac{1}{2} \ln \left(\frac{1}{w} \sqrt{\frac{J}{Q}}\right)$ in $\Phi_{d+1}$ and an additive factor of $\left(\frac{n w}{J Q}-1\right)^{-1 / 2}$ in $B_{\sigma \chi}$.

The general form of the modified solution subject to these requirements is given by

$$
\begin{aligned}
d s_{s t r, d+1}^{2} & =g_{\alpha \beta}(\rho) d \zeta^{\alpha} d \zeta^{\beta}+f_{1}(\rho) d \Omega_{d-3}^{2}+d \rho^{2} \\
\frac{1}{2} B_{\mu \nu} d x^{\mu} \wedge d x^{\nu} & =b_{\alpha \beta}(\rho) d \zeta^{\alpha} \wedge d \zeta^{\beta}+\left(\frac{n w}{J Q}-1\right)^{-1 / 2} d \sigma \wedge d \chi \\
e^{2 \Phi_{d+1}} & =\frac{1}{w} \sqrt{\frac{J}{Q}} f_{2}(\rho)
\end{aligned}
$$

where $\zeta \equiv\left(\zeta^{0}, \zeta^{1}, \zeta^{2}\right)$ stands collectively for the coordinates $\tau, \sigma$ and $\chi$, and $g_{\alpha \beta}, b_{\alpha \beta}, f_{1}$ and $f_{2}$ are some universal functions of the coordinate $\rho$, independent of any other charges and parameters. The solution in the original coordinate system, if needed, can now be found by applying the inverse of the coordinate transformation (3.19) on (3.24).

A priori we do not know the form of the functions $g_{\alpha \beta}, b_{\alpha \beta}, f_{1}$ and $f_{2}$, but let us proceed with the assumption that $\alpha^{\prime}$ corrections modify the near horizon geometry to that of an (extremal) black hole. In that case computation of the entropy requires us to integrate certain combinations of the fields over the horizon [50-55]. From the coordinate area of $4 \pi^{2} \sqrt{\frac{J n}{Q w}} \sqrt{1-\frac{J Q}{n w}}$ in the $\chi-\sigma$ plane we get a factor proportional to $\sqrt{\frac{J n}{Q w}} \sqrt{1-\frac{J Q}{n w}}$ from integration along these coordinates. The multiplicative factor of $w \sqrt{\frac{Q}{J}}$ in $e^{-2 \Phi_{d+1}}$ appears as an overall normalization factor in the $\alpha^{\prime}$ corrected effective action and gives a factor proportional to $w \sqrt{\frac{Q}{J}}$ in the entropy. Besides these multiplicative factors the contribution to the entropy cannot depend on any other charges or parameters since the solution has no non-trivial dependence on any other parameter. This gives

$$
S_{\mathrm{BH}}=C \sqrt{n w-J Q}
$$

for some constant $C$. This is in precise agreement with the answer (2.2) for the statistical entropy if we take $C=4 \pi$.

It is worth emphasizing the role of the scaling region (3.20) where the supergravity solution (3.23) is valid. As we have seen, in this region the dependence of the solution on the asymptotic moduli parameters $R_{d}$ and $g$ disappears completely. This is then used to argue that the $\alpha^{\prime}$ corrected solutions near the horizon will also be independent of these parameters. Thus this scaling region acts as a shield which isolates the near horizon region 
from the asymptotic region. However, since for large but finite charges the scaling region has a large but finite size, we expect that this shielding will work only in the leading order, and could break down at the subleading order in an expansion in inverse powers of charges. In section 1 we shall see that if we assume that the near horizon geometry has an $A d S_{2}$ factor then the independence of the entropy of the asymptotic parameters holds to all orders since there is an infinite throat region of $A d S_{2}$ that separates the near horizon geometry from the asymptotic geometry by an infinite amount.

We note in passing that if we had tried to carry out a similar scaling analysis for the rotating black hole solutions of [54] or their generalization to higher dimension, we would be led to the conclusion that the result for the black hole entropy is of the form $\sqrt{n w} g\left(J^{2} / n w\right)$ for some function $g$ [55. This is in contradiction with the microscopic result which gives the entropy to be $\sqrt{n w}$ times a function of $J / n w$. This also shows that the ring geometry is the correct geometry for describing an elementary string state with spin.

Just based on the scaling analysis we cannot determine the value of this coefficient $C$. However since from the point of view of the near horizon geometry the coordinate $\psi$ describes a non-contractible circle, we can regard this as a compact direction. In that case the solution (3.23) and its $\alpha^{\prime}$ corrected version (3.24) can be regarded as one describing the near horizon geometry of a non-rotating black hole in a space-time with $(d-1)$ noncompact dimensions, carrying $n$ units of momentum and $-w$ units of winding along the $x^{d}$ directions and $J$ units of momentum and $Q$ units of winding along the $\psi$ directions. Thus as long as non-rotating small black holes in $(d-1)$ dimensions have finite entropy, rotating $d$ dimensional black holes also have finite entropy. Furthermore if the entropy of non-rotating small black holes in $(d-1)$ dimensions agrees with the microscopic entropy, the constant $C$ is equal to $4 \pi$. This in turn will imply that the entropy of the rotating small black rings in $d$ dimension also agrees with the corresponding statistical entropy.

Regarding the solution as a $(d-1)$-dimensional small black hole also gives us some insight into the form of eq. (3.25). The $(d-1)$-dimensional effective action is known to have a continuous $\mathrm{SO}(2,2)$ symmetry to all orders in $\alpha^{\prime}$ expansion due to the fact that we are examining a sector where the fields are independent of $\sigma$ and $\chi$ directions. The only $\mathrm{SO}(2,2)$ invariant combination of the charges $n, w, J$ and $Q$ is $n w-J Q$ or a function of this combination. Since (3.25) depends on this combination we see that this formula is consistent with the $\mathrm{SO}(2,2)$ invariance of the theory.

\section{Entropy function and near horizon geometry}

In section 3 we determined the geometry of the small black ring in terms of some unknown universal functions $g_{\alpha \beta}(\rho), b_{\alpha \beta}(\rho), f_{1}(\rho)$ and $f_{2}(\rho)$. Supergravity approximation to the effective action, which is valid for large $\rho$, determines the behavior of these functions at large $\rho$. In this section we shall describe a general procedure for determining the form of these functions at small $\rho$, assuming that the near horizon geometry in this region approaches that of an extremal black hole with an $A d S_{2}$ factor, and as a result possesses an enhanced isometry $\mathrm{SO}(2,1)$. Our main tool in this analysis will be the entropy function method described in [45, 56]. 
As in section 3 we consider heterotic string theory compactified on $T^{9-d} \times S^{1}$ and consider an extremal black ring solution in this theory. From the analysis of section 3 we know that the geometry close to the horizon has two compact directions labeled by the angular coordinate $\psi$ and the coordinate along $S^{1}$ :

$$
y^{d}=\left(x^{d}-t\right) / R_{d}
$$

each with period $2 \pi$. Thus we can analyze the near horizon geometry of such a black ring by analyzing the $(d-1)$-dimensional theory obtained via dimensional reduction of the original theory on these two circles. We parametrize these $(d-1)$ dimensions by the coordinates $\left\{\xi^{m}\right\}$ with $0 \leq m \leq(d-2)$, and introduce the following $(d-1)$-dimensional fields in terms of the original $(d+1)$-dimensional fields: ${ }^{6}$

$$
\begin{aligned}
d s_{s t r, d+1}^{2}= & \widehat{G}_{m n}(\xi) d \xi^{m} d \xi^{n}+\mid ! R(\xi)^{2}\left(d y^{d}+A_{m}^{(1)}(\xi) d \xi^{m}\right)^{2}+\widetilde{R}(\xi)^{2}\left(d \psi+A_{m}^{(2)}(\xi) d \xi^{m}\right)^{2} \\
& +2 S(\xi)\left(d y^{d}+A_{m}^{(1)}(\xi) d \xi^{m}\right)\left(d \psi+A_{n}^{(2)}(\xi) d \xi^{n}\right), \\
\frac{1}{2} B_{\mu \nu} d x^{\mu} \wedge d x^{\nu}= & \frac{1}{2} \widehat{B}_{m n}(\xi) d \xi^{m} \wedge d \xi^{n}+C(\xi)\left(d y^{d}+A_{m}^{(1)}(\xi) d \xi^{m}\right) \wedge\left(d \psi+A_{m}^{(2)}(\xi) d \xi^{m}\right) \\
& +\left(d y^{d}+A_{m}^{(1)}(\xi) d \xi^{m}\right) \wedge A_{n}^{(3)}(\xi) d \xi^{n}+\left(d \psi+A_{m}^{(2)}(\xi) d \xi^{m}\right) \wedge A_{n}^{(4)}(\xi) d \xi^{n} .
\end{aligned}
$$

Thus the fields in $(d-1)$ dimensions include a metric $\widehat{G}_{m n}$, an anti-symmetric tensor field $\widehat{B}_{m n}$, four gauge fields $A_{m}^{(i)}$ for $1 \leq i \leq 4$ and five scalar fields $R, \widetilde{R}, S, C$ and $\Phi_{d+1}$. The gauge invariant field strengths constructed out of the fields $A_{m}^{(i)}$ and $\widehat{B}_{m n}$ are:

$$
\begin{aligned}
F_{m n}^{(i)} & =\partial_{m} A_{n}^{(i)}-\partial_{n} A_{m}^{(i)}, \quad i=1,2,3,4 \\
\widehat{H}_{m n p} & =\left(\partial_{m} \widehat{B}_{n p}+\text { cyclic permutations of } m, n, p\right)+\Omega_{m n p}^{\mathrm{CS}}
\end{aligned}
$$

where

$$
\Omega_{m n p}^{\mathrm{CS}}=\left\{\left(A_{m}^{(3)} F_{n p}^{(1)}+A_{m}^{(4)} F_{n p}^{(2)}\right)+\text { cyclic permutations of } m, n, p\right\}+\Omega_{m n p}^{\mathrm{CS}, \mathrm{L}}(\widehat{G}) .
$$

The action of the dimensionally reduced theory has the form:

$$
\mathcal{S}=\int d^{d-1} x \sqrt{-\operatorname{det} \widehat{G}} e^{-2 \Phi_{d+1}} \mathcal{L}_{d-1}
$$

where $\mathcal{L}_{d-1}$ is a scalar function of the scalars $R, \widetilde{R}, S$ and $C$, the metric $\widehat{G}_{m n}$, Riemann tensor, the field strengths $F_{m n}^{(i)}$ and $\widehat{H}_{m n p}, \partial_{m} \Phi_{d+1}$ and covariant derivatives of these quantities.

The presence of the Chern-Simons terms in the definition of $\widehat{H}_{m n p}$ makes this form of the action unsuitable for applying the entropy function formalism since the latter requires the Lagrangian density, when expressed in terms of the independent fields of the theory,

\footnotetext{
${ }^{6}$ Our definition of the $(d-1)$-dimensional antisymmetric tensor field $\widehat{B}_{m n}$ differs from the standard one, e.g. the one used in [1], by a term proportional to $\left(A_{[m}^{(1)} A_{n]}^{(3)}+A_{[m}^{(2)} A_{n]}^{(4)}\right)$. As a consequence the expression for the gauge Chern-Simons term appearing in the expression for $\widehat{H}_{m n p}$ is also slightly different.
} 
to be a function of manifestly covariant quantities like the metric, Riemann tensor, gauge field strengths, scalar fields, covariant derivatives of these quantities etc., and not e.g. of non-covariant quantities like the gauge fields or the spin connection. ${ }^{7}$ To get around this problem we need to dualize the theory [57]. For this note that $\widehat{H}_{m n p}$ satisfies the Bianchi identity

$$
\partial_{[m}\left(\widehat{H}_{n p q]}-\Omega_{n p q]}^{\mathrm{CS}}\right)=0 .
$$

We now introduce a new $(d-5)$-form field $\mathcal{B}_{m_{1} \ldots m_{d-5}}$, define

$$
\mathcal{H}_{m_{1} \ldots m_{d-4}}=\partial_{m_{1}} \mathcal{B}_{m_{2} \ldots m_{d-4}}+\text { cyclic permutations of } m_{1} \ldots m_{d-4} \text { with sign }
$$

to be its field strength and consider a new action

$$
\begin{gathered}
\int d^{d-1} x \sqrt{-\operatorname{det} \widehat{G}} \widetilde{\mathcal{L}}_{d-1}, \\
\sqrt{-\operatorname{det} \widehat{G}} \widetilde{\mathcal{L}}_{d-1}=\left[\sqrt{-\operatorname{det} \widehat{G}} e^{-2 \Phi_{d+1}} \mathcal{L}_{d-1}+\epsilon^{m_{1} \ldots m_{d-1}}\left(\widehat{H}_{m_{1} m_{2} m_{3}}-\Omega_{m_{1} m_{2} m_{3}}^{\mathrm{CS}}\right) \mathcal{H}_{m_{4} \ldots m_{d-1}}\right]
\end{gathered}
$$

regarding $\widehat{H}_{m n p}$ and $\mathcal{B}_{m_{1} \ldots m_{d-5}}$ as independent fields. Here $\epsilon^{m_{1} \ldots m_{d-1}}$ is totally antisymmetric in its indices, with $\epsilon^{01 \ldots(d-2)}=1$. Equations of motion of the $\mathcal{B}$ field gives the Bianchi identity (4.6). On the other hand the equations of motion of $\widehat{H}_{m_{1} m_{2} m_{3}}$ gives

$$
\frac{\delta \mathcal{S}}{\delta \widehat{H}_{m_{1} m_{2} m_{3}}}+\epsilon^{m_{1} \ldots m_{d-1}} \mathcal{H}_{m_{4} \ldots m_{d-1}}=0
$$

Together with the Bianchi identity $\partial_{\left[m_{3}\right.} \mathcal{H}_{\left.m_{4} \ldots m_{d-1}\right]}=0$, 4.10) gives us the original equations of motion of the $\widehat{B}_{m n}$ field:

$$
\partial_{p}\left(\frac{\delta \mathcal{S}}{\delta \widehat{H}_{m n p}}\right)=0 .
$$

Thus classically (4.8) and (4.5) gives rise to the same theory and we can choose to work with the action (4.8).

The action (4.8) can now be brought into a manifestly gauge, local Lorentz and general coordinate invariant form by integrating the last term in $(4.9)$ by parts. This gives a new Lagrangian density:

$$
\begin{aligned}
\sqrt{-\operatorname{det} \widehat{G}} \widetilde{\mathcal{L}}_{d-1}^{\prime}= & \sqrt{-\operatorname{det} \widehat{G}} e^{-2 \Phi_{d+1}} \mathcal{L}_{d-1} \\
& -(d-4) \epsilon^{m_{1} \ldots m_{d-1}} \partial_{m_{4}}\left(\widehat{H}_{m_{1} m_{2} m_{3}}-\Omega_{m_{1} m_{2} m_{3}}^{\mathrm{CS}}\right) \mathcal{B}_{m_{5} \ldots m_{d-1}}
\end{aligned}
$$

Since $d \Omega^{\mathrm{CS}}$ can be expressed as a function of manifestly covariant quantities like the Riemann tensor and gauge field strengths $F_{m n}^{(i)}$, the Lagrangian density $\widetilde{\mathcal{L}}_{d-1}^{\prime}$ is suitable for

\footnotetext{
${ }^{7}$ The only exception to this rule are $p$-form gauge fields whose near horizon values themselves are manifestly invariant under all the isometries of the near horizon geometry. In this case we never need to explicitly use the gauge invariance associated with these fields and can regard them as ordinary tensor fields.
} 
applying the entropy function formalism. Note however that $\widetilde{\mathcal{L}}_{d-1}^{\prime}$ does not have manifest symmetry under the gauge transformation $\mathcal{B} \rightarrow \mathcal{B}+d \Lambda, \Lambda$ being a $(d-6)$-form gauge transformation parameter. This will not affect our analysis since we shall be considering field configurations for which the field $\mathcal{B}$ (and not just its field strength $\mathcal{H}$ ) has all the necessary symmetries. Thus, as discussed in footnote 7 , we never need to make use of the gauge invariance associated with the field $\mathcal{B}$, and shall treat $\mathcal{B}$ as an independent tensor field.

We are now ready to apply the entropy function formalism. We begin with the basic postulate that in terms of the $(d-1)$-dimensional fields the near horizon metric of the extremal black ring has the structure of $A d S_{2} \times S^{d-3}$, with all other field configurations respecting the $\mathrm{SO}(2,1) \times \mathrm{SO}(d-2)$ isometry of $A d S_{2} \times S^{d-3}$. Then the general near horizon geometry of the black ring is of the form: ${ }^{8}$

$$
\begin{aligned}
\widehat{G}_{m n} d \xi^{m} d \xi^{n} & =v_{1}\left(-r^{2} d \vec{t}^{2}+\frac{d r^{2}}{r^{2}}\right)+v_{2} d \Omega_{d-3}^{2}, \\
R & =u_{R}, \quad \widetilde{R}=\widetilde{u}_{R}, \\
S & =u_{S}, \quad C=u_{C}, \quad \Phi_{d+1}=u_{\Phi}, \\
\frac{1}{2} F_{m n}^{(i)} d x^{m} \wedge d x^{n} & =e_{i} d r \wedge d \bar{t}, \quad i=1,2,3,4 \\
\frac{1}{(d-5) !} \mathcal{B}_{m_{1} \ldots m_{d-5}} d \xi^{m_{1}} \wedge \ldots \wedge d \xi^{m_{d-5}} & =\left\{\begin{array}{l}
b \text { for } d=5 \\
b d r \wedge d t \text { for } d=7, \\
0 \text { for } d \neq 5,7
\end{array}\right.
\end{aligned}
$$

where $v_{1}, v_{2}, u_{R}, \widetilde{u}_{R}, u_{S}, u_{C}, u_{\Phi}, e_{1}, e_{2}, e_{3}, e_{4}$ and $b$ are constants and $d \Omega_{d-3}$ denotes the line element on a unit $(d-3)$-sphere. Note that we have not explicitly given the $\widehat{H}$ field background; we are implicitly assuming that $\widehat{H}$ has been eliminated from the action using its equation of motion. In any case, the only possible non-zero component of $\widehat{H}_{m n p}$ consistent with the symmetries of $A d S_{2} \times S^{d-3}$ is a flux through $S^{d-3}$ in the special case of $d=6$. However since we are considering solutions without magnetic charge, - more specifically NS 5-brane charge - even for $d=6$ this flux should vanish. Thus we can consistently set $\widehat{H}_{m n p}$ to zero. The entropy function for a black ring carrying $n$ units of momentum and $-w$ units of winding along $y^{d}$ and $J$ units of momentum and $Q$ units of winding along $\psi$, is now given by 445$]^{9}$

$$
\begin{aligned}
& \mathcal{E}\left(n, J, w, Q, v_{1}, v_{2}, u_{R}, \widetilde{u}_{R}, u_{S}, u_{C}, u_{\Phi}, e_{1}, e_{2}, e_{3}, e_{4}, b\right) \\
= & 2 \pi\left(n e_{1}+J e_{2}+w e_{3}-Q e_{4}-\int_{S^{d-3}} \sqrt{-\operatorname{det} \widehat{G}} \widetilde{\mathcal{L}}_{d-1}^{\prime}\right),
\end{aligned}
$$

\footnotetext{
${ }^{8} r$ is related to the coordinate $\rho$ of section 3 by the relation $r=e^{\rho / \sqrt{v}_{1}}$. The coordinate $\bar{t}$ and $\tau$ are in general related by a scaling. However since a rescaling of the form $\bar{t} \rightarrow \lambda \bar{t}, r \rightarrow r / \lambda$, being an isometry of $A d S_{2}$, preserves the form of the solution, we can use this freedom to choose

$$
\bar{t}=\tau, \quad r=c e^{\rho / \sqrt{v}_{1}},
$$

for some constant $c$.

${ }^{9}$ According to the convention of section 3, $n, J, w$ and $-Q$ represent the charges associated with the $A_{m}^{(1)}, A_{m}^{(2)}, A_{m}^{(3)}$ and $A_{m}^{(4)}$ fields respectively. This explains the signs in front of various charges in 4.14. Note however that we have chosen to call $-w$ and $Q$ the winding charges along $y^{d}$ and $\psi$ respectively.
} 
where $\sqrt{-\operatorname{det} \widehat{G}} \widetilde{\mathcal{L}}_{d-1}^{\prime}$ has to be evaluated on the horizon. The entropy is given by $\mathcal{E}$ after extremizing it with respect to the near horizon parameters $v_{1}, v_{2}, u_{R}, \widetilde{u}_{R}, u_{S}, u_{C}, u_{\Phi}, e_{1}$, $e_{2}, e_{3}, e_{4}$ and $b$.

This gives a general algebraic procedure for determining the near horizon geometry of a small black ring for a given action. We shall now show that the entropy calculated from this formalism has the same dependence on $n, w, J$ and $Q$ as was derived in the previous section. For this we need to use the known scaling properties of the $\alpha^{\prime}$ corrected tree level effective action of the heterotic string theory. First of all note from (4.12) that

$$
\widetilde{\mathcal{L}}_{d-1}^{\prime} \rightarrow \lambda{\widetilde{\mathcal{L}_{d-1}^{\prime}}}^{\prime} \quad \text { under } \quad e^{-2 \Phi_{d+1}} \rightarrow \lambda e^{-2 \Phi_{d+1}}, \quad \mathcal{B}_{m_{1} \ldots m_{d-5}} \rightarrow \lambda \mathcal{B}_{m_{1} \ldots m_{d-5}}
$$

The freedom of changing the periodicity along the circle $S^{1}$ labeled by $y^{d}$ and subsequently making a rescaling of the $y^{d}$ coordinate to bring the period back to $2 \pi$ gives another scaling property of the Lagrangian density:

$$
\begin{array}{rlrl}
\widetilde{\mathcal{L}}_{d-1}^{\prime} \rightarrow \kappa{\widetilde{\mathcal{L}_{d-1}^{\prime}}}^{\prime} \quad \text { under } \quad A_{m}^{(1)} & \rightarrow \kappa^{-1} A_{m}^{(1)}, \quad A_{m}^{(3)} \rightarrow \kappa A_{m}^{(3)}, \\
R & \rightarrow \kappa R, & S \rightarrow \kappa S, \\
C & \rightarrow \kappa C .
\end{array}
$$

Finally there is a similar scaling property associated with the scaling of the $\psi$ coordinate. This gives

$$
\begin{array}{rlrl}
\widetilde{\mathcal{L}}_{d-1}^{\prime} \rightarrow \eta \widetilde{\mathcal{L}}_{d-1}^{\prime} \quad \text { under } \quad A_{m}^{(2)} & \rightarrow \eta^{-1} A_{m}^{(2)}, & A_{m}^{(4)} & \rightarrow \eta A_{m}^{(4)}, \\
\widetilde{R} & \rightarrow \eta \widetilde{R}, & S \rightarrow \eta S, \\
C & \rightarrow \eta C .
\end{array}
$$

From (4.15), 4.16) and (4.17) we can derive the following properties of the entropy function:

$$
\begin{array}{rlrl}
\mathcal{E} \rightarrow \lambda \mathcal{E} \quad \text { under } \quad e^{-2 u_{\Phi}} & \rightarrow \lambda e^{-2 u_{\Phi},}, \quad b \rightarrow \lambda b, & Q \rightarrow \lambda Q, \\
n & \rightarrow \lambda n, & w & \rightarrow \lambda w, \quad J \rightarrow \lambda J, \\
\mathcal{E} \rightarrow \kappa \mathcal{E} \quad \text { under } e_{1} & \rightarrow \kappa^{-1} e_{1}, \quad n \rightarrow \kappa^{2} n, \quad J \rightarrow \kappa J, \\
e_{3} & \rightarrow \kappa e_{3}, & Q & \rightarrow \kappa Q, \quad u_{R} \rightarrow \kappa u_{R}, \\
u_{S} & \rightarrow \kappa u_{S}, & u_{C} & \rightarrow \kappa u_{C},
\end{array}
$$

and

$$
\begin{aligned}
\mathcal{E} \rightarrow \eta \mathcal{E} \quad \text { under } \quad n & \rightarrow \eta n, \quad e_{2} \rightarrow \eta^{-1} e_{2}, \quad J \rightarrow \eta^{2} J \\
w & \rightarrow \eta w, \quad e_{4} \rightarrow \eta e_{4}, \quad \widetilde{u}_{R} \rightarrow \eta u_{R}, \\
u_{S} & \rightarrow \eta u_{S}, \quad u_{C} \rightarrow \eta u_{C} .
\end{aligned}
$$

From this it follows that after elimination of the various near horizon parameters by extremizing $\mathcal{E}$, the entropy $S_{\mathrm{BH}}=\mathcal{E}$ has the property:

$$
\begin{array}{llll}
S_{\mathrm{BH}} \rightarrow \lambda S_{\mathrm{BH}} & \text { under } \quad n \rightarrow \lambda n, \quad w \rightarrow \lambda w, \quad J \rightarrow \lambda J \quad Q \rightarrow \lambda Q, \\
S_{\mathrm{BH}} \rightarrow \kappa S_{\mathrm{BH}} \quad \text { under } \quad n \rightarrow \kappa^{2} n, \quad Q \rightarrow \kappa Q, \quad J \rightarrow \kappa J,
\end{array}
$$


and

$$
S_{\mathrm{BH}} \rightarrow \eta S_{\mathrm{BH}} \quad \text { under } \quad n \rightarrow \eta n, \quad w \rightarrow \eta w, \quad J \rightarrow \eta^{2} J .
$$

eqs. (4.21), (4.21) and 4.22) give

$$
S_{\mathrm{BH}}=\sqrt{n w} f\left(\frac{J Q}{n w}\right)
$$

for some function $f$.

We can constrain the form of the function $f$ by noting that the dimensionally reduced Lagrangian density has a further symmetry induced by a rotation in the $y^{d}-\psi$ plane by the matrix

$$
U=\left(\begin{array}{cc}
\cos \theta & \sin \theta \\
-\sin \theta & \cos \theta
\end{array}\right)
$$

This induces a transformation

$$
\left(\begin{array}{cc}
R^{2} & S \\
S & R^{2}
\end{array}\right) \rightarrow U\left(\begin{array}{cc}
R^{2} & S \\
S & \widetilde{R}^{2}
\end{array}\right) U^{T}, \quad\left(\begin{array}{c}
A_{m}^{(1)} \\
A_{m}^{(2)}
\end{array}\right) \rightarrow U\left(\begin{array}{c}
A_{m}^{(1)} \\
A_{m}^{(2)}
\end{array}\right), \quad\left(\begin{array}{c}
A_{m}^{(3)} \\
A_{m}^{(4)}
\end{array}\right) \rightarrow U\left(\begin{array}{c}
A_{m}^{(3)} \\
A_{m}^{(4)}
\end{array}\right) .
$$

Thus the entropy function $\mathcal{E}$ is invariant under

$$
\begin{gathered}
\left(\begin{array}{c}
e_{1} \\
e_{2}
\end{array}\right) \rightarrow U\left(\begin{array}{c}
e_{1} \\
e_{2}
\end{array}\right), \quad\left(\begin{array}{c}
e_{3} \\
e_{4}
\end{array}\right) \rightarrow U\left(\begin{array}{l}
e_{3} \\
e_{4}
\end{array}\right), \quad\left(\begin{array}{l}
n \\
J
\end{array}\right) \rightarrow U\left(\begin{array}{l}
n \\
J
\end{array}\right), \\
\left(\begin{array}{c}
w \\
-Q
\end{array}\right) \rightarrow U\left(\begin{array}{c}
w \\
-Q
\end{array}\right), \quad\left(\begin{array}{ll}
u_{R}^{2} & u_{S} \\
u_{S} & \widetilde{u}_{R}^{2}
\end{array}\right) \rightarrow U\left(\begin{array}{ll}
u_{R}^{2} & u_{S} \\
u_{S} & \widetilde{u}_{R}^{2}
\end{array}\right) U^{T} .
\end{gathered}
$$

As a result the black hole entropy $S_{\mathrm{BH}}$ is invariant under

$$
\left(\begin{array}{c}
w \\
-Q
\end{array}\right) \rightarrow U\left(\begin{array}{c}
w \\
-Q
\end{array}\right), \quad\left(\begin{array}{l}
n \\
J
\end{array}\right) \rightarrow U\left(\begin{array}{l}
n \\
J
\end{array}\right) .
$$

Together with (4.23) this uniquely fixes the form of $S_{\mathrm{BH}}$ to be

$$
S_{\mathrm{BH}}=C \sqrt{n w-J Q},
$$

for some constant $C$.

If the entropy function had no flat directions then we would also be able to determine the near horizon parameters labeling the solution by demanding that the solution remains invariant under the symmetry transformations described above. This however is not possible due to the existence of two flat directions of the entropy function. ${ }^{10}$ Thus

\footnotetext{
${ }^{10}$ If we consider the case where the coordinates $y^{d}$ and $\psi$ are both compact and there are no fluxes then the vacuum moduli space, ignoring the $T^{9-d}$ factor, is $\mathrm{SO}(2,2) /(\mathrm{SO}(2) \times \mathrm{SO}(2))$ where $\mathrm{SO}(2,2)$ represents the continuous T-duality symmetry associated with a $T^{2}$ compactification, and $\mathrm{SO}(2) \times \mathrm{SO}(2)$ is its maximal compact subgroup. Since the charge vector $(n, J, w, Q)$ is invariant under an $\operatorname{SO}(2,1)$ subgroup of this $\mathrm{SO}(2,2)$ group, once we switch on a flux proportional to this charge vector the moduli space of solutions becomes the two dimensional space $\mathrm{SO}(2,1) / \mathrm{SO}(2)$. This would correspond to two flat directions of the entropy function. The only exceptions are light-like charge vectors in which case the little group will be $\mathrm{SO}(1,1)$, but this corresponds to the case $n w-J Q=0$ which we are not considering here. In general a symmetry transformation of the type given in eqs. (4.15)-(4.17) and (4.25), instead of leaving the solution unchanged, will take one solution to another solution.
} 
for a given set of charges $(n, J, w, Q)$ the entropy function extremization condition gives a two parameter family of solutions. Which of these solutions actually appear as the near horizon configuration of the black ring will depend on the asymptotic data - in particular the asymptotic values of the moduli fields and the information that we are considering a black ring in $d$-dimensions rather than a black hole in $(d-1)$ dimensions where the $\psi$ direction remains compact even asymptotically. Thus the entropy function itself cannot give complete information about the near horizon geometry even if we knew the full $\alpha^{\prime}$ corrected action.

On the other hand, the analysis in section 3 does know about the asymptotic infinity since it is based on a solution of the supergravity equations of motion embedded in an asymptotically flat $d$-dimensional spacetime. Therefore the solution $(3.23)$ in the scaling region (3.20) does not involve any unknown parameter, and in turn completely determines the general form (3.24) of the solution near the horizon. This suggests that we can combine the results of section 3 and this section to fix the form of the near horizon geometry. We first note that the solution (4.13) corresponds to the $(d+1)$-dimensional field configuration:

$$
\begin{aligned}
d s_{s t r, d+1}^{2}= & v_{1}\left(-r^{2} d \bar{t}^{2}+\frac{d r^{2}}{r^{2}}\right)+v_{2} d \Omega_{d-3}^{2}+u_{R}^{2}\left(d y^{d}+e_{1} r d \bar{t}\right)^{2}+\widetilde{u}_{R}^{2}\left(d \psi+e_{2} r d \bar{t}\right)^{2} \\
& +2 u_{S}\left(d y^{d}+e_{1} r d \bar{t}\right)\left(d \psi+e_{2} r d \bar{t}\right), \\
\frac{1}{2} B_{\mu \nu} d x^{\mu} \wedge d x^{\nu}= & u_{C}\left(d y^{d}+e_{1} r d \bar{t}\right) \wedge\left(d \psi+e_{2} r d \bar{t}\right)+e_{3} r\left(d y^{d}+e_{1} r d \bar{t}\right) \wedge d \bar{t} \\
& +e_{4} r\left(d \psi+e_{2} r d \bar{t}\right) \wedge d \bar{t} \\
e^{-2 \Phi_{d+1}}= & e^{-2 u_{\Phi}}
\end{aligned}
$$

In writing (4.29) we have used the coordinate transformations $y^{d} \rightarrow y^{d}+a \bar{t}, \chi \rightarrow \chi+b \bar{t}$ to remove the constant terms in $A_{t}^{(1)}$ and $A_{t}^{(2)}$. On the other hand (3.19), (3.24) tells us that if we use the $(\sigma, \chi)$ coordinate system defined through

$$
\sigma=\sqrt{\frac{n}{w}-\frac{J Q}{w^{2}}} y^{d}, \quad \chi=\sqrt{\frac{J}{Q}} w+\frac{\sqrt{J Q}}{w} y^{d},
$$

then the solution has a universal form except for some additive constants in $B_{\sigma \chi}$ and $\ln \Phi_{d+1}$. Requiring (4.29) to satisfy this requirement we get the form of the solution to be:

$$
\begin{aligned}
d s_{s t r, d+1}^{2}= & c_{1}\left(-r^{2} d \bar{t}^{2}+\frac{d r^{2}}{r^{2}}\right)+c_{2} d \Omega_{d-3}^{2}+c_{3}\left(d \sigma+c_{4} r d \bar{t}\right)^{2} \\
& +c_{5}\left(d \chi+c_{6} r d \bar{t}\right)^{2}+c_{7}\left(d \sigma+c_{4} r d \bar{t}\right)\left(d \chi+c_{6} r d \bar{t}\right) \\
e^{2 \Phi_{d+1}}= & c_{8} \sqrt{\frac{J}{Q}} \frac{1}{w} \\
\frac{1}{2} B_{\mu \nu} d x^{\mu} \wedge d x^{\nu}= & c_{9} r d \sigma \wedge d \bar{t}+c_{10} r d \chi \wedge d \bar{t}+c_{11} d \sigma \wedge d \chi+\left(\frac{n w}{J Q}-1\right)^{-1 / 2} d \sigma \wedge d \chi,
\end{aligned}
$$

where $c_{1}, \ldots, c_{11}$ are some numerical constants independent of any charges or other asymptotic data, which can in principle be determined, up to the flat direction, by extremizing 
the entropy function. This gives the general form of the solution close to the horizon. Since the periodicities of the $\sigma$ and $\chi$ directions, as given in (3.21), depends on the charges, the solution has some additional implicit dependence on the charges besides the ones shown in (4.31). This can be made explicit by rewriting the solution in terms of $\left(y^{d}, \psi, \bar{t}, r\right)$ coordinate system using (4.30) such that both the compact coordinates $y^{d}$ and $\psi$ have period $2 \pi$.

In the spirit of the discussion at the end of section 3 we note that from the point of view of the near horizon geometry the coordinate $\psi$ can be regarded as a compact direction. In that case the entropy function $\mathcal{E}$ considered here can be regarded as that of a $(d-1)$ dimensional non-rotating black hole carrying $n$ units of momentum and $-w$ units of winding along the $y^{d}$ direction and $J$ units of momentum and $Q$ units of winding along the $\psi$ direction. Thus as long as non-rotating small black holes in $(d-1)$-dimension have finite entropy, rotating $d$-dimensional black holes also have finite entropy. ${ }^{11}$ Furthermore if the entropy of non-rotating small black holes in $(d-1)$ dimension agrees with the microscopic entropy, the constant $C$ is equal to $4 \pi$. This in turn will imply that the entropy of the rotating small black rings in $d$ dimension also agrees with the corresponding statistical entropy.

In this context it is also worth emphasizing that if we were studying a small black hole with four charges in $(d-1)$ dimensions instead of a ring in $d$-dimensions, then the near horizon geometry does contain two arbitrary parameters whose values need to be determined from the knowledge of the asymptotic values of the moduli field. In particular if we carry out an analysis analogous to that of section 3, we shall find that even in the scaling region the supergravity solution continues to depend on a pair of asymptotic moduli.

\section{Discussion}

In this paper we studied the near-horizon geometry of the small black ring carrying charge quantum numbers $(n, J, w, Q)$ from two different viewpoints. First we examined the full singular black ring solution of the supergravity theory describing a rotating spinning string and identified a scaling region where, in a suitable coordinate system, the solution ceased to depend on the asymptotic moduli and its dependence on the various charges appear in a fashion such that higher derivative corrections are insensitive to the charges. This allowed us to express the $\alpha^{\prime}$-corrected solution in terms of a set of universal functions independent of any parameters. The entropy computed from the $\alpha^{\prime}$-corrected solution was found to have the form $C \sqrt{n w-J Q}$ where $C$ is a numerical constant that cannot be computed in the absence of a complete knowledge of all the $\alpha^{\prime}$ corrections. Even if the $\alpha^{\prime}$ correction to the effective action is known, in this approach it would be a highly non-trivial task to actually find the solution to the equations of motion and calculate the coefficient $C$ from the $\alpha^{\prime}$ corrected action. Nevertheless the result for the entropy found in this approach is consistent with the result for the statistical entropy of the same system, given by $4 \pi \sqrt{n w-J Q}$.

\footnotetext{
${ }^{11}$ For example if we add to the action a $(d-1)$-dimensional Gauss-Bonnet term then the resulting nonrotating small black holes acquire a finite entropy [18].
} 
In the second approach we focussed our attention on the near horizon geometry instead of examining the full solution. Assuming that the near horizon geometry has an enhanced $\mathrm{SO}(2,1)$ symmetry besides the manifest rotational isometries of the solution, we can write down the general form of the solution in terms of a set of constant parameters, and the entropy is obtained by extremizing the entropy function with respect to these parameters. Using the various known scaling properties of the $\alpha^{\prime}$-corrected effective action we then determined the dependence of the entropy obtained this way on the charges, and arrived at the same answer $C \sqrt{n w-J Q}$ for some constant $C$. Again computation of the constant $C$ requires knowledge of the $\alpha^{\prime}$-corrected action, but in this case once we know the action there is a simple algorithm to compute the entropy without having to solve any differential equations.

In principle extremization of the entropy function also determines the parameters characterizing the near horizon geometry. In practice however this is plagued by the problem that the entropy function relevant for this problem has two flat directions, and hence the extremization condition does not determine the solution uniquely. Thus which member of this two parameter family appears as the actual near horizon geometry depends on the asymptotic data. Nevertheless by combining the information about the asymptotic data from the first approach with the requirement of enhanced $\mathrm{SO}(2,1)$ symmetry of the near horizon geometry we can determine the near horizon geometry in terms of the charges and a few (presently unknown) numerical constants.

Clearly the most important open problem is to find the constant $C$. An insight into this problem can be gained from the observation that this constant is the same as what appears in the expression for the entropy of a non-rotating small black hole in one less dimension. Thus agreement between the microscopic and macroscopic entropy for non-rotating small black hole in $(d-1)$-dimension would also imply agreement between microscopic and macroscopic entropy of a rotating small black ring in $d$-dimensions. At present however concrete analysis of this constant $C$ has been performed only in the case of four dimensional small black holes [31. Initial studies of these black holes were based on keeping only a small subset of higher derivative corrections to the effective action, e.g. the F-type terms [9] or the Gauss-Bonnet term [18], yielding the same answer $C=4 \pi$. However later a general procedure for analyzing these black holes was developed by Kraus and Larsen [19] where, based on the assumption that the $A d S_{2}$ and the $S^{1}$ factor of the near horizon geometry combine to form an $A d S_{3}$ space, they were able to relate the coefficient $C$ to the coefficients of the gauge and gravitational Chern-Simons terms in the action. Since these coefficients are known exactly, $C$ also can be calculated exactly. This yielded the same answer $C=4 \pi$. In principle it should be possible to generalize the results of Kraus and Larsen to higher dimensional black holes, but so far this has not been done.

\section{Acknowledgments}

N.I. would like to thank all his friends in India, especially the string theory group at TIFR, where he shared really fantastic and fun time together with the people there by discussing string theory, and where part of this work was done. M.S. would like to thank TIFR and 
MIT CTP where part of this work was done. A.D., N.I. and M.S. would also like to thank the Aspen Center for Physics where part of this work was done. The work of N.I. was supported in part by the National Science Foundation under Grant No. PHY99-07949. M.S. was supported in part by Department of Energy grant DE-FG03-92ER40701 and a Sherman Fairchild Foundation postdoctoral fellowship.

\section{A. Supergravity small black rings in general dimensions}

In this appendix we shall construct small black ring solutions in heterotic string theory with $d$ non-compact space-time dimensions, describing a rotating elementary string. The same solution in a different U-duality frame was derived in [43] as supergravity supertubes.

Consider heterotic string theory in $\mathbf{R}_{t} \times \mathbf{R}^{d-1} \times S^{1} \times T^{9-d}$ with $4 \leq d \leq 9$. We denote the coordinates of $\mathbf{R}_{t}, \mathbf{R}^{d-1}$ and $S^{1}$ by $t, \mathbf{x}=\left(x^{1}, \ldots, x^{d-1}\right)$ and $x^{d}$ respectively, and the coordinate radius of the $x^{d}$ direction by $R_{d}$. We would like to study the geometry of a small black ring sitting in the noncompact $d$-dimensional space $\mathbf{R}_{t} \times \mathbf{R}^{d-1}$. As in section 3, we shall regard this as a solution in the $(d+1)$-dimensional theory obtained by dimensional reduction of the ten dimensional heterotic string theory on $T^{9-d}$. Thus we shall use the $(d+1)$-dimensional fields to express the solution. This in particular will require us to take the solution to be independent of the coordinates of $T^{9-d}$; this is done by 'smearing' the ten dimensional solution along $T^{9-d}$.

In 44, 44, a large class of supergravity solutions were derived which correspond to a fundamental string with an arbitrary left-moving traveling wave on it, $\mathbf{x}=\mathbf{F}\left(t-x^{d}\right)$, where $\mathbf{F}=\left(F_{1}, \ldots, F_{d-1}\right)$ are arbitrary functions. In [48] (see also [36]), the situation where a fundamental string is wrapping $-w(w \gg 1)$ times around the $x^{d}$ direction and carrying $n(n \gg 1)$ units of momentum along the $x^{d}$ direction was considered in the particular case of $d=5$. It was argued there that in such a situation the supergravity description is obtained by smearing the solution of 44 in the $x^{d}$ direction. The smeared solution can be compactified on the $x^{d}$ direction, giving a five-dimensional solution with an arbitrary profile of the fundamental string, $\mathbf{x}=\mathbf{F}(v)$, in the noncompact $\mathbf{R}^{4}$.

This construction of [48] can be straightforwardly generalized to arbitrary $d$. Namely, a solution of the $(d+1)$-dimensional supergravity equations of motion, describing a fundamental string wrapping $-w(w \gg 1)$ times around the $x^{d}$ direction, carrying $n(n \gg 1)$ units of momentum along the $x^{d}$ direction, and having an arbitrary shape in the noncompact $\mathbf{R}^{d-1}$ direction parametrized by the profile function $\mathbf{x}=\mathbf{F}(v)(0 \leq v \leq L)$, is given by:

$$
\begin{aligned}
d s_{s t r, d+1}^{2} & =f_{f}^{-1}\left[-\left(d t-A_{i} d x^{i}\right)^{2}+\left(d x^{d}-A_{i} d x^{i}\right)^{2}+\left(f_{p}-1\right)\left(d t-d x^{d}\right)^{2}\right]+d \mathbf{x}_{d-1}^{2} \\
e^{2 \Phi_{d+1}} & =g^{2} f_{f}^{-1} \\
B_{t d} & =-\left(f_{f}^{-1}-1\right), \\
B_{t i} & =-B_{d i}=f_{f}^{-1} A_{i},
\end{aligned}
$$


where $i=1,2, \ldots, d-1$, and ${ }^{12}$

$$
\begin{aligned}
& f_{f}(\mathbf{x})=1+\frac{Q_{f}}{L} \int_{0}^{L} \frac{d v}{|\mathbf{x}-\mathbf{F}(v)|^{d-3}}, \\
& f_{p}(\mathbf{x})=1+\frac{Q_{f}}{L} \int_{0}^{L} \frac{|\dot{\mathbf{F}}(v)|^{2} d v}{|\mathbf{x}-\mathbf{F}(v)|^{d-3}}, \\
& A_{i}(\mathbf{x})=-\frac{Q_{f}}{L} \int_{0}^{L} \frac{\dot{F}_{i}(v) d v}{|\mathbf{x}-\mathbf{F}(v)|^{d-3}} .
\end{aligned}
$$

The dot denotes derivative with respect to $v$, and $L=2 \pi w R_{d}$. We also define

$$
Q_{p} \equiv \frac{Q_{f}}{L} \int_{0}^{L}|\dot{\mathbf{F}}(v)|^{2} d v .
$$

For large $|\mathbf{x}|, f_{f}-1$ and $f_{p}-1$ fall off as $Q_{f} /|\mathbf{x}|^{d-3}$ and $Q_{p} /|\mathbf{x}|^{d-3}$ respectively. By computing the flux of the gauge fields associated with $G_{d \mu}$ and $B_{d \mu}$ at infinity, one finds that the relations between $Q_{f}, Q_{p}$ and quantized charges $n, w$ are

$$
Q_{f}=\frac{16 \pi G_{d} R_{d}}{(d-3) \Omega_{d-2} \alpha^{\prime}} w, \quad Q_{p}=\frac{16 \pi G_{d}}{(d-3) \Omega_{d-2} R_{d}} n
$$

where $\Omega_{D}$ is the area of $S^{D}$ and $G_{d}$ is the $d$-dimensional Newton constant:

$$
16 \pi G_{d}=\frac{16 \pi G_{d+1}}{2 \pi R_{d}}=\frac{(2 \pi)^{d-3} g^{2} \alpha^{\prime(d-1) / 2}}{R_{d}} .
$$

In order to arrive at (A.4), (A.5) we have used the fact that in the absence of higher derivative corrections, which are irrelevant in the asymptotic region, the action has the form given in (3.1)-(3.3). By examining the asymptotic form of the metric and the results of 58 one also sees that the angular momentum associated with the solution in the $x^{i}-x^{j}$ plane is given by

$$
J_{i j}=\frac{(d-3) \Omega_{d-2}}{16 \pi G_{d}} \frac{Q_{f}}{L} \int_{0}^{L}\left(F_{i} \dot{F}_{j}-F_{j} \dot{F}_{i}\right) d v .
$$

Before considering the small black ring solution, let us first consider the case with a circular profile:

$$
\mathbf{F}=\mathbf{F}^{(0)}, \quad\left\{\begin{array}{l}
F_{1}^{(0)}+i F_{2}^{(0)}=R e^{i \omega v}, \\
F_{3}^{(0)}=\cdots=F_{d-1}^{(0)}=0,
\end{array} \quad \omega=\frac{2 \pi Q}{L}=\frac{Q}{w R_{d}} .\right.
$$

This corresponds to a fundamental string which winds $Q$ times along the ring of radius $R$ in the $x^{1}-x^{2}$ plane 48, 59. Introducing the coordinate system $(s, \psi, w, \vec{\xi})$ by

$$
\begin{aligned}
& x^{1}=s \cos \psi, \quad x^{2}=s \sin \psi, \\
& x^{3}=w \xi^{1}, \quad x^{4}=w \xi^{2}, \quad \ldots, \quad x^{d-1}=w \xi^{d-3} \quad \sum_{a=1}^{d-3}\left(\xi^{a}\right)^{2}=1,
\end{aligned}
$$

\footnotetext{
${ }^{12}$ The relation to the harmonic functions in 48, 36] is $f_{f}=H^{-1}, f_{p}=K+1$.
} 
the harmonic functions in (A.2) are computed as

$$
\begin{aligned}
f_{f} & =1+Q_{f}\left(s^{2}+w^{2}+R^{2}\right)^{-\frac{d-3}{2}}{ }_{2} F_{1}\left(\frac{d-3}{4}, \frac{d-1}{4} ; 1 ; \frac{4 R^{2} s^{2}}{\left(s^{2}+w^{2}+R^{2}\right)^{2}}\right), \\
f_{p} & =1+Q_{p}\left(s^{2}+w^{2}+R^{2}\right)^{-\frac{d-3}{2}}{ }_{2} F_{1}\left(\frac{d-3}{4}, \frac{d-1}{4} ; 1 ; \frac{4 R^{2} s^{2}}{\left(s^{2}+w^{2}+R^{2}\right)^{2}}\right), \\
A_{\psi} & =-\left(\frac{d-3}{2}\right) q R^{2} s^{2}\left(s^{2}+w^{2}+R^{2}\right)^{-\frac{d-1}{2}}{ }_{2} F_{1}\left(\frac{d-1}{4}, \frac{d+1}{4} ; 2 ; \frac{4 R^{2} s^{2}}{\left(s^{2}+w^{2}+R^{2}\right)^{2}}\right),
\end{aligned}
$$

where we have defined

$$
q \equiv Q_{f} \omega
$$

and ${ }_{2} F_{1}(\alpha, \beta ; \gamma ; z)$ denotes the hypergeometric function. For odd $d$, the hypergeometric functions in (A.9) can be written as rational functions, while for even $d$ they involve elliptic integrals. Furthermore, from (A.3),

$$
Q_{p}=Q_{f} R^{2} \omega^{2}
$$

Using (A.10) and the last equation of (A.7), one finds

$$
q=\frac{16 \pi G_{d}}{(d-3) \Omega_{d-2} \alpha^{\prime}} Q
$$

Moreover, from (A.6) one finds that the solution carries an angular momentum $J=Q R^{2} / \alpha^{\prime}$ in the $x^{1}-x^{2}$ plane. This gives

$$
R^{2}=\alpha^{\prime} \frac{J}{Q}
$$

From (A.4, (A.11), A.13), and the last equation of (A.7), we obtain

$$
J Q=n w,
$$

i.e., the circular configuration (A.7) saturates the Regge bound.

Now let us proceed to construct the small black ring solution. This can be done by taking the profile function to be 49

$$
\mathbf{F}=\mathbf{F}^{(0)}+\delta \mathbf{F},
$$

where $\delta \mathbf{F}$ describes fluctuations around $\mathbf{F}^{(0)}$, whose detailed form is irrelevant as long as it satisfies certain conditions to be explained below. As the simplest example, take $\delta \mathbf{F}$ to be

$$
\delta F_{1}+i \delta F_{2}=a e^{i(\nu v+b)}
$$

where we eventually take the limit

$$
\frac{a}{R} \rightarrow 0, \quad \frac{\nu}{\omega} \rightarrow \infty, \quad a \nu: \text { fixed }
$$


In other words, $\delta \mathbf{F}$ of (A.16) represents a very small-amplitude $(a \ll R)$, high-frequency $(\nu \gg \omega)$ fluctuation. Because of the first condition in (A.17), $\mathbf{F}$ in the denominators in the integrand of (A.2) can be replaced by $\mathbf{F}^{(0)}$. Now expand this denominator as

$$
\begin{aligned}
\left|\mathbf{x}-\mathbf{F}^{(0)}\right|^{-(d-3)} & =\left[s^{2}+w^{2}+R^{2}-2 s R \cos (\omega v-\psi)\right]^{-\frac{d-3}{2}} \\
& =\left(s^{2}+w^{2}+R^{2}\right)^{-\frac{d-3}{2}} \sum_{k=0}^{\infty} \frac{1}{k !} \frac{\Gamma\left(\frac{d-3}{2}+k\right)}{\Gamma\left(\frac{d-3}{2}\right)}\left[\frac{2 s R \cos (\omega v-\psi)}{s^{2}+w^{2}+R^{2}}\right]^{k} .
\end{aligned}
$$

On the other hand, in the numerator, e.g. for $f_{p}$, we have

$$
|\dot{\mathbf{F}}|^{2}=\left|\dot{\mathbf{F}}^{(0)}+\delta \dot{\mathbf{F}}\right|^{2}=R^{2} \omega^{2}+a^{2} \nu^{2}+2 R \omega a \nu \cos [(\omega-\nu) v-b] .
$$

When we multiply (A.19) and (A.19), and integrate it over $v$, there will be nonvanishing contributions only when the frequencies of the cosines in (A.19) and (A.19) cancel each other, which happens only for $k \gtrsim \frac{\nu}{\omega}$. Since $\frac{\nu}{\omega} \rightarrow \infty$ in the limit (A.17), in fact there is no contribution from the last term in (A.19). Similarly, there is no contribution from $\delta \mathbf{F}$ to $A_{i}$ in A.2); only $\mathbf{F}^{(0)}$ contributes.

At the end of the day, the only effect of introducing the fluctuation (A.16) is to change eq. (A.11) to

$$
Q_{p}=Q_{f}\left(R^{2} \omega^{2}+a^{2} \nu^{2}\right),
$$

whereas other expressions (A.9), (A.12) and (A.13) are unchanged. These give the supergravity small black ring solution we were after. Note that eqs. (A.4), (A.20), (A.13), and the last equation of (A.7) now imply the Regge bound,

$$
J Q<n w .
$$

Even if one considers more complicated fluctuations than (A.16) by taking linear combinations of many modes in all the $x^{i}$ directions $(1 \leq i \leq(d-1))$, the above results remain unchanged as long as the condition (A.17) is met for each mode, except that the $a^{2} \nu^{2}$ term in (A.20) will be replaced by a sum over the contribution from all the modes. The fact that the resulting solution (A.9) is insensitive to the precise form of the fluctuation $\delta \mathbf{F}$ is the reflection of the fact that this supergravity small black ring represents all the underlying microstates whose entropy is given by (1.3).

Although in (A.9) we presented the small black ring solution in the $(s, \psi, w, \vec{\xi})$ coordinate system, it is more convenient for the analysis in the main text to go to the coordinate system $(y, \psi, x, \vec{\xi})$ defined by

$$
s=\frac{\sqrt{y^{2}-1}}{x-y} R, \quad w=\frac{\sqrt{1-x^{2}}}{x-y} R, \quad-1 \leq x \leq 1, \quad-\infty<y \leq-1 .
$$

In terms of these coordinates, the harmonic functions (A.9) become

$$
\begin{aligned}
f_{f} & =1+\frac{Q_{f}}{R^{d-3}}\left(\frac{x-y}{-2 y}\right)^{(d-3) / 2}{ }_{2} F_{1}\left(\frac{d-3}{4}, \frac{d-1}{4} ; 1 ; 1-\frac{1}{y^{2}}\right), \\
f_{p} & =1+\frac{Q_{p}}{R^{d-3}}\left(\frac{x-y}{-2 y}\right)^{(d-3) / 2}{ }_{2} F_{1}\left(\frac{d-3}{4}, \frac{d-1}{4} ; 1 ; 1-\frac{1}{y^{2}}\right), \\
A_{\psi} & =-\left(\frac{d-3}{2}\right) \frac{q}{R^{d-5}} \frac{\left(y^{2}-1\right)(x-y)^{(d-5) / 2}}{(-2 y)^{(d-1) / 2}}{ }_{2} F_{1}\left(\frac{d-1}{4}, \frac{d+1}{4} ; 2 ; 1-\frac{1}{y^{2}}\right),
\end{aligned}
$$


and the $(d-1)$-dimensional flat metric $d \mathbf{x}_{d-1}^{2}$ can be written as

$$
d \mathbf{x}_{d-1}^{2}=\frac{R^{2}}{(x-y)^{2}}\left[\frac{d y^{2}}{y^{2}-1}+\left(y^{2}-1\right) d \psi^{2}+\frac{d x^{2}}{1-x^{2}}+\left(1-x^{2}\right) d \Omega_{d-4}^{2}\right] .
$$

Eqs. (A.1), (A.23) and (A.24), together with the definitions of various parameters given in (A.4), (A.5), (A.12) and (A.13), describe the supergravity small black ring solution. Note that once the solution has been obtained this way, we can forget about how it was constructed, and simply analyze its properties by treating this as a singular solution of the supergravity equations of motion. This is the view point we have adopted in section 3.

\section{References}

[1] J.M. Maldacena, A. Strominger and E. Witten, Black hole entropy in M-theory, JHEP 12 (1997) 002 hep-th/9711053.

[2] K. Behrndt et al., Higher-order black-hole solutions in $N=2$ supergravity and Calabi-Yau string backgrounds, Phys. Lett. B 429 (1998) 289 hep-th/9801081.

[3] G. Lopes Cardoso, B. de Wit and T. Mohaupt, Corrections to macroscopic supersymmetric black-hole entropy, Phys. Lett. B 451 (1999) 309 hep-th/9812082.

[4] G. Lopes Cardoso, B. de Wit and T. Mohaupt, Deviations from the area law for supersymmetric black holes, Fortschr. Phys. 48 (2000) 49 hep-th/9904005.

[5] T. Mohaupt, Black hole entropy, special geometry and strings, Fortschr. Phys. 49 (2001) 3 hep-th/0007195.

[6] G. Lopes Cardoso, B. de Wit and T. Mohaupt, Macroscopic entropy formulae and non-holomorphic corrections for supersymmetric black holes, Nucl. Phys. B 567 (2000) 87 hep-th/9906094.

[7] G. Lopes Cardoso, B. de Wit, J. Kappeli and T. Mohaupt, Stationary BPS solutions in $N=2$ supergravity with $R^{2}$ interactions, JHEP 12 (2000) 019 hep-th/0009234.

[8] G. Lopes Cardoso, B. de Wit, J. Kappeli and T. Mohaupt, Examples of stationary BPS solutions in $N=2$ supergravity theories with $R^{2}$-interactions, Fortschr. Phys. 49 (2001) 557 hep-th/0012232.

[9] A. Dabholkar, Exact counting of black hole microstates, Phys. Rev. Lett. 94 (2005) 241301 hep-th/0409148.

[10] A. Dabholkar, R. Kallosh and A. Maloney, A stringy cloak for a classical singularity, JHEP 12 (2004) 059 hep-th/0410076.

[11] A. Sen, How does a fundamental string stretch its horizon?, JHEP 05 (2005) 059 hep-th/0411255.

[12] V. Hubeny, A. Maloney and M. Rangamani, String-corrected black holes, JHEP 05 (2005) 035 hep-th/0411272.

[13] K. Behrndt, G. Lopes Cardoso and S. Mahapatra, Exploring the relation between $4 D$ and $5 D$ BPS solutions, Nucl. Phys. B 732 (2006) 200 hep-th/0506251.

[14] A. Dabholkar and J.A. Harvey, Nonrenormalization of the superstring tension, Phys. Rev. Lett. 63 (1989) 478. 
[15] A. Sen, Extremal black holes and elementary string states, Mod. Phys. Lett. A 10 (1995) 2081 hep-th/9504147.

[16] A.W. Peet, Entropy and supersymmetry of D-dimensional extremal electric black holes versus string states, Nucl. Phys. B 456 (1995) 732 hep-th/9506200.

[17] A. Sen, Black holes and elementary string states in $N=2$ supersymmetric string theories, JHEP 02 (1998) 011 hep-th/9712150.

[18] A. Sen, Stretching the horizon of a higher dimensional small black hole, JHEP 07 (2005) 073 hep-th/0505122.

[19] P. Kraus and F. Larsen, Microscopic black hole entropy in theories with higher derivatives, JHEP 09 (2005) 034 hep-th/0506176.

[20] P. Kraus and F. Larsen, Holographic gravitational anomalies, JHEP 01 (2006) 022 hep-th/0508218.

[21] P. Kraus, Lectures on black holes and the $A d S_{3} / C F T(2)$ correspondence, hep-th/0609074.

[22] A. Sen, Black holes, elementary strings and holomorphic anomaly, JHEP 07 (2005) 063 hep-th/0502126.

[23] A. Dabholkar, F. Denef, G.W. Moore and B. Pioline, Exact and asymptotic degeneracies of small black holes, JHEP 08 (2005) 021 hep-th/0502157.

[24] A. Dabholkar, F. Denef, G.W. Moore and B. Pioline, Precision counting of small black holes, JHEP 10 (2005) 096 hep-th/0507014.

[25] I. Bena and P. Kraus, Microscopic description of black rings in AdS/CFT, JHEP 12 (2004) 070 hep-th/0408186.

[26] I. Bena and P. Kraus, Microstates of the D1-D5-KK system, Phys. Rev. D 72 (2005) 025007 hep-th/0503053.

[27] D. Gaiotto, A. Strominger and X. Yin, New connections between $4 D$ and 5D black holes, JHEP 02 (2006) 024 hep-th/0503217.

[28] H. Elvang, R. Emparan, D. Mateos and H.S. Reall, Supersymmetric 4 D rotating black holes from 5D black rings, JHEP 08 (2005) 042 hep-th/0504125.

[29] D. Gaiotto, A. Strominger and X. Yin, 5D black rings and 4D black holes, JHEP 02 (2006) 023 hep-th/0504126.

[30] I. Bena, P. Kraus and N.P. Warner, Black rings in Taub-NUT, Phys. Rev. D 72 (2005) 084019 hep-th/0504142.

[31] N. Iizuka and M. Shigemori, A note on D1-D5-J system and 5D small black ring, JHEP 08 (2005) 100 hep-th/0506215.

[32] H. Elvang, R. Emparan, D. Mateos and H.S. Reall, A supersymmetric black ring, Phys. Rev. Lett. 93 (2004) 211302 hep-th/0407065.

[33] I. Bena and N.P. Warner, One ring to rule them all ... and in the darkness bind them?, Adv. Theor. Math. Phys. 9 (2005) 667 hep-th/0408106.

[34] H. Elvang, R. Emparan, D. Mateos and H.S. Reall, Supersymmetric black rings and three-charge supertubes, Phys. Rev. D 71 (2005) 024033 hep-th/0408120. 
[35] J.P. Gauntlett and J.B. Gutowski, General concentric black rings, Phys. Rev. D 71 (2005) 045002 hep-th/0408122.

[36] S.D. Mathur, The fuzzball proposal for black holes: an elementary review, Fortschr. Phys. 53 (2005) 793 hep-th/0502050.

[37] S.D. Mathur, The quantum structure of black holes, Class. and Quant. Grav. 23 (2006) R115 hep-th/0510180.

[38] O. Lunin and S.D. Mathur, Statistical interpretation of Bekenstein entropy for systems with a stretched horizon, Phys. Rev. Lett. 88 (2002) 211303 hep-th/0202072.

[39] J.G. Russo and L. Susskind, Asymptotic level density in heterotic string theory and rotating black holes, Nucl. Phys. B 437 (1995) 611 hep-th/9405117.

[40] B.C. Palmer and D. Marolf, Counting supertubes, JHEP 06 (2004) 028 hep-th/0403025.

[41] D. Marolf and B.C. Palmer, Gyrating strings: a new instability of black strings?, Phys. Rev. D 70 (2004) 084045 hep-th/0404139.

[42] D. Bak, Y. Hyakutake, S. Kim and N. Ohta, A geometric look on the microstates of supertubes, Nucl. Phys. B 712 (2005) 115 hep-th/0407253.

[43] R. Emparan, D. Mateos and P.K. Townsend, Supergravity supertubes, JHEP 07 (2001) 011 hep-th/0106012.

[44] A. Dabholkar, J.P. Gauntlett, J.A. Harvey and D. Waldram, Strings as solitons and black holes as strings, Nucl. Phys. B 474 (1996) 85 hep-th/9511053.

[45] A. Sen, Black hole entropy function and the attractor mechanism in higher derivative gravity, JHEP 09 (2005) 038 hep-th/0506177.

[46] A. Dabholkar, N. Iizuka, A. Iqubal and M. Shigemori, Precision microstate counting of small black rings, Phys. Rev. Lett. 96 (2006) 071601 hep-th/0511120.

[47] C.G. Callan Jr., J.M. Maldacena and A.W. Peet, Extremal black holes as fundamental strings, Nucl. Phys. B 475 (1996) 645 hep-th/9510134.

[48] O. Lunin and S.D. Mathur, Metric of the multiply wound rotating string, Nucl. Phys. B 610 (2001) 49 hep-th/0105136.

[49] V. Balasubramanian, P. Kraus and M. Shigemori, Massless black holes and black rings as effective geometries of the D1-D5 system, Class. and Quant. Grav. 22 (2005) 4803 hep-th/0508110.

[50] R.M. Wald, Black hole entropy in the Noether charge, Phys. Rev. D 48 (1993) 3427 gr-qc/9307038.

[51] T. Jacobson, G. Kang and R.C. Myers, On black hole entropy, Phys. Rev. D 49 (1994) 6587 gr-qc/9312023.

[52] V. Iyer and R.M. Wald, Some properties of Noether charge and a proposal for dynamical black hole entropy, Phys. Rev. D 50 (1994) 846 gr-qc/9403028.

[53] T. Jacobson, G. Kang and R.C. Myers, Black hole entropy in higher curvature gravity, gr-qc/9502009.

[54] A. Sen, Black hole solutions in heterotic string theory on a torus, Nucl. Phys. B 440 (1995) 421 hep-th/9411187. 
[55] A. Dabholkar, N. Iizuka, A. Iqubal, A. Sen and M. Shigemori, unpublished.

[56] D. Astefanesei, K. Goldstein, R.P. Jena, A. Sen and S.P. Trivedi, Rotating attractors, JHEP 10 (2006) 058 hep-th/0606244.

[57] B. Sahoo and A. Sen, Alpha' corrections to extremal dyonic black holes in heterotic string theory, JHEP 01 (2007) 010 [hep-th/0608182].

[58] R.C. Myers and M.J. Perry, Black holes in higher dimensional space-times, Ann. Phys. (NY) $172(1986) 304$.

[59] O. Lunin, J.M. Maldacena and L. Maoz, Gravity solutions for the D1-D5 system with angular momentum, hep-th/0212210. 